\title{
Flow Starvation Mitigation for Wireless Mesh Networks
}

\author{
Keivan Ronasi, Sathish Gopalakrishnan, and Vincent W.S. Wong \\ Department of Electrical and Computer Engineering \\ The University of British Columbia, Vancouver, Canada \\ e-mail: $\{$ keivanr, sathish, vincentw $\} @$ ece.ubc.ca
}

\begin{abstract}
Wireless mesh networks can provide scalable highspeed Internet access at a low cost. Fair channel access among different nodes in the wireless mesh network, however, is an important consideration that needs technological solutions before mesh networks can be widely deployed. Lack of fairness significantly decreases the throughput of nodes that are more than one hop away from mesh gateways. We propose an analytical model and use simulation studies to establish the existence of starvation in mesh networks even when we can ameliorate problems due to exposed terminals. Motivated by the inability of standard medium access control (MAC) protocols to limit starvation, we propose a modification to the MAC protocol to alleviate flow starvation. Our proposed algorithm improves the channel usage of short-term flows with nodes that are multiple hops from the gateway by a factor of 7 in some cases with a penalty of $20 \%$ reduction in total throughput across all nodes. Our proposed algorithm also has a better performance than two other schemes in terms of a higher fairness index.
\end{abstract}

\section{INTRODUCTION}

Wireless mesh networks (WMNs) are being deployed to provide low-cost high-bandwidth Internet access on a large scale. In a WMN, stationary wireless mesh nodes (or routers) provide network connectivity for mobile wireless mesh clients. Multiple wireless mesh nodes are interconnected to form a wireless backbone. Several mesh nodes also serve as the gateways (GW), which connect to the Internet. The mesh architecture, in which all mesh nodes participate in creating an infrastructure for decentralized data transmission, makes a WMN a stable and economically viable solution for providing universal Internet access.

In most WMN usage scenarios, the available bandwidth must be utilized in a fair manner by all mesh nodes. Unfortunately, the carrier sense multiple access with collision avoidance (CSMA/CA) medium access control (MAC) protocol in IEEE 802.11 can lead to unfairness in multi-hop data transmission in WMNs. In addition, in the transport layer, the transmission control protocol (TCP) does not account for fairness among users and sometimes may lead to starvation of some nodes depending upon the location of nodes and their distance from the GW. An undesirable condition is the flow starvation which can cause some flows to attain extremely low data transfer rates compared to other flows.

Starvation in wireless networks has been observed in some earlier studies. The poor performance of CSMA/CA-based MAC protocols has been considered through simulations and analytical modeling [1]-[3]. It has been shown that when senders observe different channel conditions compared to their neighbors, some of them can encounter flow starvation. The existence of unfairness in single hop networks, where any two communicating nodes are within transmission range of each other, was reported in [4]. The work in [5] classified various two-flow scenarios into twelve classes and investigated the short-term and long-term performance in each class. Similar studies for multi-hop networks can be found in [6]-[8].

The work in [9] identified bi-stability as a cause for starvation in WMNs. In a linear topology, bi-stability occurs when nodes closer to the GW enter a phase of successful packet transmissions that forces other nodes to wait for an extended period of time. The work in [9] is closely related to our work in this paper. They analytically determined the existence of starvation in a multi-hop network with linear topology, which they called the basic topology. Analytical modeling was carried out for the two-node scenario but not for the three-node scenario. No consideration was given to possible solutions to the exposed terminal problem. Furthermore, they considered the interference model where nodes that are two hops away are outside each other's transmission range and carrier sensing range. Also, another work which is closely related to our work is Idle Sense [10], which adjusts the contention window of the IEEE 802.11 MAC protocol to achieve fairness in wireless local area networks (WLANs). Idle Sense, however, is suitable for single hop WLANs only and does not consider the effect of data flows with multiple hops.

In [11] and [12], it has been argued that modifications to the transport layer can provide fairness despite unfair MAC protocols. Multi-channel protocols have also been considered to mitigate starvation [13], as well as rate control techniques [14], multi-path solutions [15], and optimization-based approaches [16]. All these methods are relevant but we believe that solutions at the MAC layer are central to performance enhancement and we suggest improvements at the MAC layer to limit starvation.

In this paper, we establish the existence of, and analyze the extent of, starvation in two-hop and three-hop scenarios where a node is at most two or three hops from the GW. Specifically, we consider the interference model where some adjacent nodes are within carrier sensing range but are not within transmission range of each other. This is a common situation when a WMN is employed to provide network connectivity in dense environments such as a university campus. The contributions 
of this paper are as follows:

1) We propose a simplified Markov chain model to analyze WMNs with linear topology and numerically compute the degree of unfairness between nodes.

2) We propose a fair binary exponential backoff (FBEB) algorithm to reduce the extent of starvation. Our proposed algorithm uses the intuition that, to improve fairness, mesh nodes that have successfully transmitted a data packet should not be permitted to eagerly transmit more data packets. By delaying the transmission of successive packets we are able to reduce the degree of starvation. This effect is achieved by adapting the contention window for transmissions in the IEEE 802.11 protocol.

3) Our analytical results show that solving the exposed terminal problem alone is insufficient for combatting starvation in WMNs.

4) Simulation results also show that our proposed FBEB algorithm can improve the data rate of starving nodes by a factor of 7 in some cases. Fairness is achieved by limiting the aggressive use of the channel; the overall loss in throughput across all nodes was observed to be about $20 \%$. The loss in overall throughput is, however, acceptable if fairness is a key parameter in WMNs. We also show through our simulations that our proposed FBEB algorithm outperforms other schemes [9], [10] under the 2-hop carrier sense assumption.

Our work presumes a linear topology (or a chain) for mesh nodes. This is an elementary structure and understanding the behavior of nodes arranged in such a topology provides insight into the behavior of larger networks, which could be studied as a collection of chains.

This paper is organized as follows. The Markov chain model for studying the flow starvation among nodes is described in Section II. Our proposed algorithm is presented in Section III. The analytical and simulation results are presented in Section IV. Conclusions are given in Section V.

\section{Analytical Modeling of Flow Starvation}

In this section, we present an analytical model to study the effect of flow starvation among nodes in WMNs with a linear topology. We first define the state space and then describe how to determine the state transition probabilities.

\section{A. System Model}

We consider a linear topology such that each node is within the transmission range of its neighboring nodes (see Fig. 1). For nodes (or wireless mesh routers) that are two hops away from each other, they are within the carrier sensing range but not within each other's transmission range.

We consider the Transmission Control Protocol at the transport layer. Nodes that send data packets receive TCP acknowledgments (ACKs). The number of data packets sent by a node before receiving an ACK is less than or equal to the TCP congestion window parameter, $c g w$, which we assume to be fixed for ease of analysis. We assume that nodes are always backlogged. A source node can attempt to send data packets

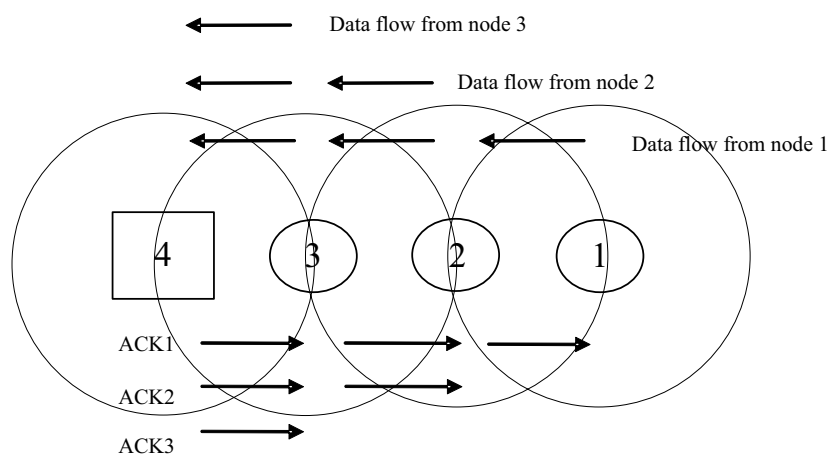

Fig. 1. Upstream data transmission in a linear topology with three wireless mesh routers $\{1,2,3\}$ and a gateway $(\mathrm{GW})\{4\}$.

as long as the number of unacknowledged packets is less than $c g w$. Any intermediate node may either have data packets to send in the upstream direction (from node to GW) or ACKs to send in the downstream direction (from GW to the node).

In the data link layer, each node listens to the channel before attempting a packet transmission. If the channel is busy, the node continuously listens to the channel until it senses that the channel is idle. The node then randomly selects a slot among the next $C W$ slots (where $C W$ is the contention window size) and transmits a packet in that slot if the channel is idle. Packet collision can happen when two neighboring nodes transmit a packet in the same slot. When packet collision occurs, the corresponding nodes double their contention window and start the sending process again. If the contention window reaches its maximum value, it is reset and the packet to be sent is being dropped.

\section{B. States and Transition Probabilities}

We model the state of each node using a tuple that consists of the number of packets in each queue: a separate queue is maintained for data packets from a particular flow and a separate queue is utilized for ACKs corresponding to a particular flow. Furthermore, the state of each node also includes the size of its contention window.

Let $\mathcal{N}=\{1,2, \ldots,|\mathcal{N}|\}$ denote the set of nodes (i.e., wireless mesh routers and GW). In the linear topology, node $|\mathcal{N}|$ is $\mathrm{GW}$, node $n \in\{1,2, \ldots,|\mathcal{N}|-1\}$ is the $(|\mathcal{N}|-n)^{\text {th }}$ hop downstream neighbor of GW. Thus, node 1 is the farthest node from $\mathrm{GW}$ in terms of the number of hops. Let vector $\boldsymbol{c}=\left(C W_{1}, C W_{2}, \ldots, C W_{|\mathcal{N}|}\right)$, where $C W_{n}$ is the contention window size of node $n \in \mathcal{N}$. Let $\mathcal{F}$ denote the set of flows. A flow $f \in \mathcal{F}$ corresponds to a source and destination pair. For $n \in \mathcal{N}$, we let vector $\boldsymbol{q}_{n}^{d}=\left(Q_{n, 1}^{d}, Q_{n, 2}^{d}, \ldots, Q_{n,|\mathcal{F}|}^{d}\right)$, where $Q_{n, f}^{d}$ denotes the number of data packets stored in node $n$ for flow $f \in \mathcal{F}$. Similarly, for $n \in \mathcal{N}$, we let vector $\boldsymbol{q}_{n}^{a}=\left(Q_{n, 1}^{a}, Q_{n, 2}^{a}, \ldots, Q_{n,|\mathcal{F}|}^{a}\right)$, where $Q_{n, f}^{a}$ denotes the number of ACK packets stored in node $n$ for flow $f \in \mathcal{F}$.

The system state is an aggregate state that is composed of the states of individual nodes. The system state is defined as

$$
\boldsymbol{s}:=\left(\boldsymbol{c}, \boldsymbol{q}_{n}^{d}, \boldsymbol{q}_{n}^{a}, \forall n \in \mathcal{N}\right) .
$$


TABLE I

Transmission Probabilities of Different Events in a Linear Topology With One GW $\left(n_{4}\right)$ and Three Mesh Routers $\left(n_{3}, n_{2}, n_{1}\right)$.

\begin{tabular}{|c|c|}
\hline Event type & Probability \\
\hline $\begin{array}{l}\text { One successful data transmission. } \\
\text { Node } n \in \mathcal{N} \text { sends data packet for flow } f \in \mathcal{F} \text {. }\end{array}$ & $\begin{array}{l}\left(Q_{n, f}^{d} / Q_{n}^{d}\right) P_{n}^{d}\left(1-P_{n-1}^{d}-P_{n-1}^{a}\right)\left(1-P_{n+1}^{d}-P_{n+1}^{a}\right) \\
\times\left(1-P_{n-2}^{d}-P_{n-2}^{a}\right)\left(1-P_{n+2}^{d}-P_{n+2}^{a}\right)\end{array}$ \\
\hline $\begin{array}{l}\text { One successful ACK transmission. } \\
\text { Node } n \in \mathcal{N} \text { sends an ACK packet for flow } f \in \mathcal{F} \text {. }\end{array}$ & $\begin{array}{l}\left(Q_{n, f}^{a} / Q_{n}^{a}\right) P_{n}^{a}\left(1-P_{n-1}^{d}-P_{n-1}^{a}\right)\left(1-P_{n+1}^{a}-P_{n+1}^{d}\right) \\
\times\left(1-P_{n-2}^{d}-P_{n-2}^{a}\right)\left(1-P_{n+2}^{d}-P_{n+2}^{a}\right)\end{array}$ \\
\hline $\begin{array}{l}\text { Two successful transmissions. } n_{2} \text { sends an ACK for flow } f_{1} \in \mathcal{F} \text {. } \\
n_{3} \text { sends a data packet for flow } f_{2} \in \mathcal{F} \text {. Node } n_{3} \text { is downstream } \\
\text { neighbor of GW } n_{4} \text {. Node } n_{2} \text { is downstream neighbor of } n_{3} \text {. }\end{array}$ & $\begin{array}{l}\left(Q_{n_{2}, f_{1}}^{a} Q_{n_{3}, f_{2}}^{d}\right) /\left(Q_{n_{2}}^{a} Q_{n_{3}}^{d}\right) \\
\times P_{n_{2}}^{a}\left(1-P_{n_{1}}^{d}-P_{n_{1}}^{a}\right) P_{n_{3}}^{d}\left(1-P_{n_{4}}^{d}-P_{n_{4}}^{a}\right)\end{array}$ \\
\hline Two data packets transmission. One successful transmission. & $\begin{array}{l}P_{m}^{d} P_{n}^{d} \prod_{i \neq m, n}\left(1-P_{i}^{d}-P_{i}^{a}\right) \\
\text { where number of hops between nodes } m \text { and } n \leq 2 \text {. }\end{array}$ \\
\hline One successful transmission and two nodes in collision. & $\left(P_{n_{1}}^{d}+P_{n_{1}}^{a}\right)\left(P_{n_{2}}^{d}+P_{n_{2}}^{a}\right) P_{n_{3}}^{d}\left(1-P_{n_{4}}^{d}-P_{n_{4}}^{a}\right)$ \\
\hline $\begin{array}{l}\text { Collision between two nodes. } \\
(m \text { is a downstream neighbor for } n \text { ) }\end{array}$ & $\begin{array}{l}P_{m}^{d} P_{n}^{a} \prod_{i \neq m, n}\left(1-P_{i}^{d}-P_{i}^{a}\right) \\
\text { where number of hops between nodes } m \text { and } n \leq 2 .\end{array}$ \\
\hline Collision between three nodes. & $\left(P_{n_{1}}^{d}+P_{n_{1}}^{a}\right)\left(P_{n_{2}}^{d}+P_{n_{2}}^{a}\right) P_{n_{3}}^{a}\left(1-P_{n_{4}}^{d}-P_{n_{4}}^{a}\right)$ \\
\hline Collision between all four nodes. & $\prod_{n_{i} \in \mathcal{N}}\left(P_{n_{i}}^{d}+P_{n_{i}}^{a}\right)$ \\
\hline Idle. & $\mathcal{N}\left(1-P_{n_{i}}^{d}-P_{n_{i}}^{a}\right)$ \\
\hline
\end{tabular}

Let $\mathcal{S}$ denote the set of feasible states and $|\mathcal{S}|$ denote the total number of feasible states. Depending on the system state $s \in \mathcal{S}$, each node has a specific contention state and a specific queue state. The current system state may trigger several events and these events may cause the system to transition to a different state. The number of data packets waiting to be sent from node $n \in \mathcal{N}$ is

$$
Q_{n}^{d}=\sum_{f \in \mathcal{F}} Q_{n, f}^{d} .
$$

The number of ACK packets that are waiting to be sent from node $n$ is

$$
Q_{n}^{a}=\sum_{f \in \mathcal{F}} Q_{n, f}^{a} .
$$

The probability that node $n \in \mathcal{N}$ sends a data packet when the channel is free is

$$
P_{n}^{d}(s)=\left(\frac{Q_{n}^{d}}{Q_{n}^{a}+Q_{n}^{d}}\right)\left(\frac{2}{C W_{n}}\right)
$$

and the probability that node $n$ sends an ACK packet is

$$
P_{n}^{a}(s)=\left(\frac{Q_{n}^{a}}{Q_{n}^{a}+Q_{n}^{d}}\right)\left(\frac{2}{C W_{n}}\right) .
$$

In our model, all feasible events, including one or more successful transmissions from different nodes, any combination of simultaneous successful and unsuccessful transmissions, collisions between two or more nodes, and an idle event in which no node transmits are considered. State transition probabilities are calculated based on $P_{n}^{d}(\boldsymbol{s})$ and $P_{n}^{a}(\boldsymbol{s})$ and the equations are listed in Table I. In the second and third rows in Table $\mathrm{I}$, the notations $n+i$ and $n-i$ denote the nodes that are $i$ hops upstream and downstream, respectively, from node $n$. We set $P_{n}^{d}=P_{n}^{a}=0$ if $n \notin \mathcal{N}$. Given (4), (5), and Table I, the system evolution can be described by a Markov chain with an $|\mathcal{S}|$ by $|\mathcal{S}|$ state transition matrix $\Phi$.

\section{Channel Usage}

Let $\pi(s)$ denote the steady state probability that the system is in state $s \in \mathcal{S}$. Let row vector $\pi=(\pi(s), \forall s \in \mathcal{S})$. Using the state transition probability matrix $\Phi$, we can determine $\pi$ by solving the following equations

$$
\pi=\pi \Phi,
$$

and

$$
\boldsymbol{\pi} \mathbf{1}=1,
$$

where 1 is an $|\mathcal{S}|$ by 1 unit vector.

For each flow $f \in \mathcal{F}$, we define an $|\mathcal{S}|$ by $|\mathcal{S}|$ binary matrix $R_{f}$ where each row corresponds to a feasible current state and each column corresponds to a feasible next state. An element in matrix $R_{f}$ is equal to 1 when the transition from the current state to the next state leads to the reception of an ACK by the source of flow $f$, and is equal to 0 otherwise. The ratio of channel usage for flow $f \in \mathcal{F}$, denoted as $p_{f}$, is

$$
p_{f}=\boldsymbol{\pi}\left(R_{f} \odot \Phi\right) \mathbf{1},
$$

where $\odot$ represents the Hadamard product operator of two matrices.

\section{FAIR BINARY EXPONENTIAL BACKOFF (FBEB) ALGORITHM}

A primary reason for flow starvation is that CSMA/CAbased MAC protocols permit a node which succeeds in transmitting a data packet to hold the channel for many consecutive time slots. When nodes encounter a packet collision event, they have to increase their contention window in order to reduce the chance of further collisions in future. Suppose a node $n$ has successfully transmitted its data packet, it resets its contention window. This will allow node $n$ to send its data packets more aggressively and prevent other nodes from accessing the channel. Other contending nodes can send their 




Fig. 2. Fair Binary Exponential Backoff (FBEB) algorithm.

data packets either after node $n$ has drained its packet queue or when they have selected an idle time slot, with low probability, to transmit a packet.

The limitation of the CSMA/CA protocol design is that nodes reset their contention window after the first successful transmission. The contention window can be used to control access to the channel. When many flows compete for the channel, we can ensure that successful nodes retain a higher contention window in order to increase the channel access probability for other nodes. This cautiousness with regard to the contention window can improve fairness at the expense of a slight loss in aggregate throughput.

The scheme that we propose, at its core, can be described as follows. A node which successfully transmits a data packet does not reset its contention window immediately. Instead this node will defer, for duration $T$, resetting the contention window to understand the extent to which other nodes are competing for the channel. The waiting time, $T$, depends on the level of previously known contention in the network, and the size of the contention window is a reasonable estimate of the level of contention. We therefore set $T$ to be proportional to the contention window. Specifically, for each node $n \in \mathcal{N}$, the value of $T$ is selected from a uniform distribution over the range $\left(0, T_{f w}\right)$ where $T_{f w}=\overline{C W_{n}} / 6$ and $\overline{C W_{n}}$ is the average contention window size of node $n$. This range is obtained empirically as being the suitable value for $T_{f w}$.

When data traffic load is small, few nodes are competing for access to the channel and the contention level is low. A successful node may, however, never reset its contention window if it does not observe another transmission during the waiting period. To prevent this situation, any node which has $K_{\text {succ }}$ successful consecutive transmissions must reset its contention window. Via simulations, we recommend the value of $K_{\text {succ }}$ to be equal to 4 .

We modify the backoff scheme in the IEEE 802.11 MAC protocol; we call our proposed scheme the Fair Binary Exponential Backoff Algorithm (see Algorithm 1). Fig. 2 illustrates the behavior of the FBEB algorithm with two nodes.

\section{Performance Evaluation And Comparison}

In this section, we first present the analytical results for the upstream scenario for two cases: a) with exposed terminals, and b) with a solution [17] to the exposed terminal problem.

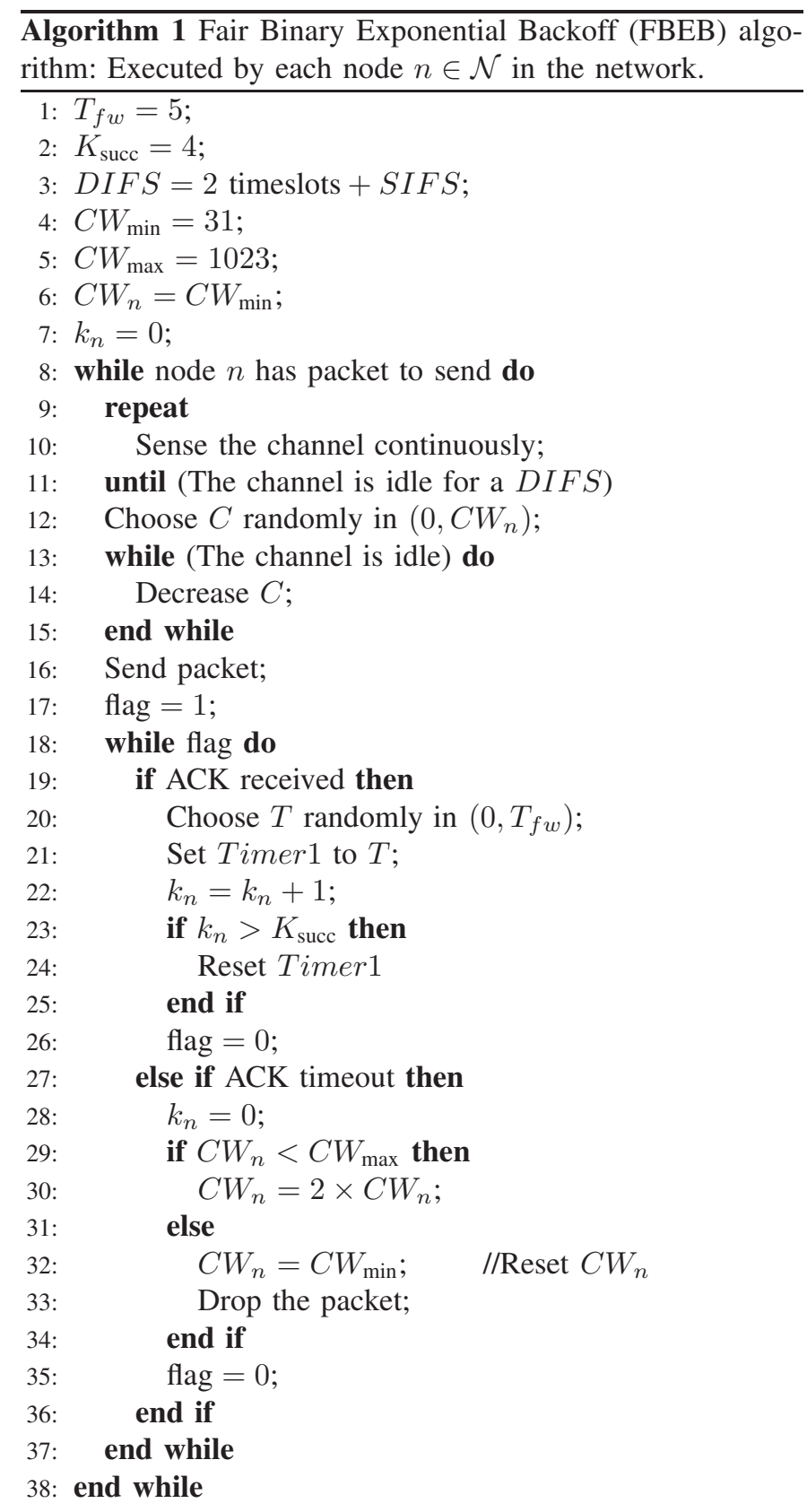

Timer 1 Reset Procedure:

1: $C W_{n}=C W_{\min } ; \quad / / \operatorname{Reset} C W_{n}$

We then present the simulation results of our proposed FBEB algorithm and compare it with counter starvation policy (CSP) [9] and Idle Sense [10] schemes in terms of throughput and fairness.

\section{A. Results from Analytical Model}

By using standard computational methods for Markov chains, we obtain the steady state probabilities for successful transmission at different nodes in the 3-hop upstream scenario. We also determine the relative channel usage ratio for data transmission of each flow by dividing its success probability 


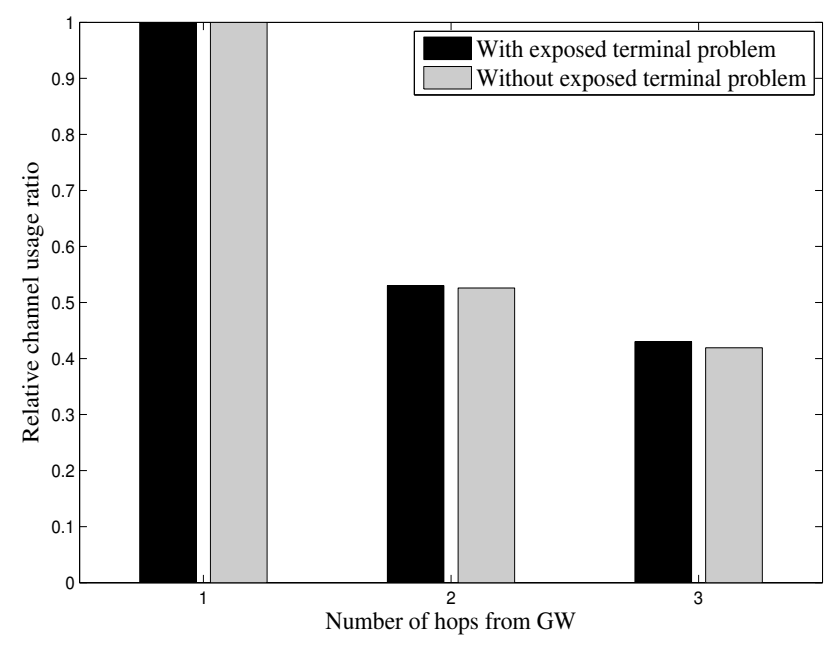

Fig. 3. Analytical modeling for upstream scenario with and without exposed terminal. Solving the exposed terminal problem does not reduce flow starvation.

by the flow with the highest success probability. We use our analytical model to study the effect of flow starvation. A practical approach is proposed in [17] which can eliminate (or reduce) the exposed terminal problem. In 3-hop chains, where exposed terminals exist, we examine the benefits of eliminating exposed terminals on starvation in a WMN. Two adjacent mesh nodes can send data packets if the receiver of each transmission is not within the transmission range of the other sender. Results in Fig. 3 show that solving the exposed terminal problem does not alleviate starvation, and that better scheduling decisions need to be made to reduce unfair channel access.

\section{B. Performance of FBEB Algorithm}

We evaluate the performance of our proposed FBEB algorithm via simulation and compare it with two other schemes: counter starvation policy (CSP) [9] and Idle Sense [10]. For the parameters used in the simulation model, nodes are 48 $\mathrm{m}$ away from each other. The transmission range and carrier sense range are chosen to be 50 and $100 \mathrm{~m}$, respectively. The minimum contention window for the IEEE 802.11 MAC protocol is set at 31 . The TCP NewReno is used in the transport layer. For generating traffic, we simulate the file transfer protocol (FTP) on top of TCP.

Fig. 4 (a) and (b) show the performance comparison results for the short-term $(90 \mathrm{sec})$ and long-term $(500 \mathrm{sec})$ scenarios, respectively. For the case of short-term flows (see Fig. 4 (a)), FBEB improves the data rate for the node that is farthest from the GW by a factor of 7. Although the fairness is improved significantly, it comes at an expense of $20 \%$ loss in aggregate throughput between FBEB algorithm and the unmodified IEEE 802.11 MAC protocol, which attains the highest aggregate throughput among the four schemes. For the case of longterm flows (see Fig. 4 (b)), the ratio of throughput obtained from FBEB algorithm to 802.11 MAC in the third node is 1.12 and the reduction in aggregate throughput is $5 \%$. Thus, (a)

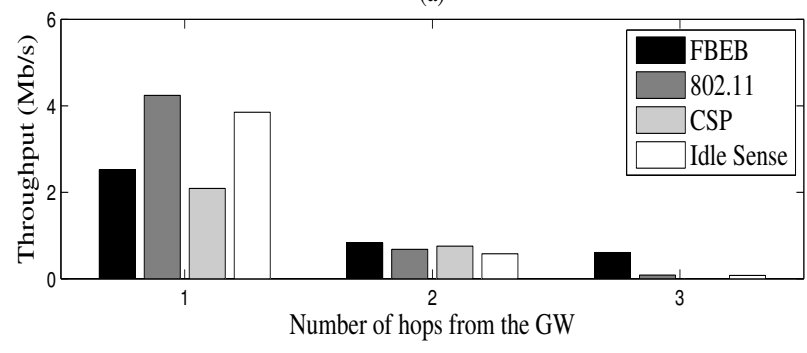

(b)

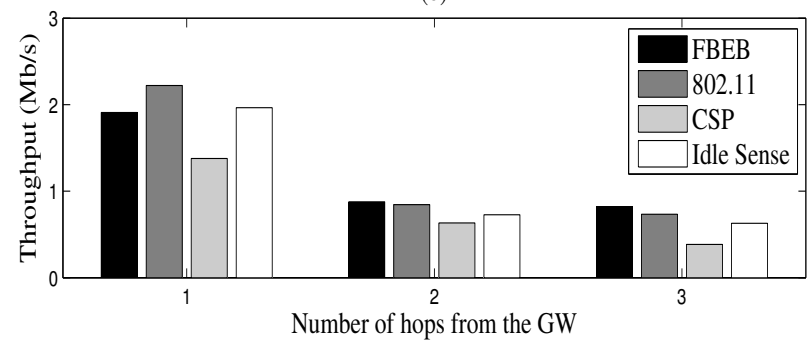

Fig. 4. Comparison between different schemes. Our proposed FBEB algorithm improves fairness at the cost of a drop in overall throughput. FBEB, in fact, leads to higher fairness when compared with Idle Sense [10], CSP [9] and the unmodified IEEE 802.11 MAC. (a) Short-term starvation where FBEB improves the data rate for the flow from the farthest node significantly. (b) Improvement on the long-term behavior where the gains are relatively modest.

TABLE II

ACHIEVED FAIRnESS AMONG DIFFERENT FLOWS

\begin{tabular}{|c|c|c|}
\hline \multirow{2}{*}{ Algorithm } & \multicolumn{2}{|c|}{ Fairness Index } \\
\cline { 2 - 3 } & $\begin{array}{c}\text { Short-term } \\
(90 \mathrm{sec})\end{array}$ & $\begin{array}{c}\text { Long-term } \\
(500 \mathrm{sec})\end{array}$ \\
\hline FBEB & 0.7044 & 0.8526 \\
\hline CSP [9] & 0.5458 & 0.7823 \\
\hline Idle Sense [10] & 0.4461 & 0.7682 \\
\hline
\end{tabular}

our proposed FBEB algorithm is an effective scheme for shortterm flows.

We also use the Jain's fairness index [18] to quantitatively measure the fairness of the throughput attained among different flows under various algorithms. Let $\Psi$ denote Jain's fairness index. We have

$$
\Psi=\frac{\left(\sum_{f \in \mathcal{F}} x_{f}\right)^{2}}{|\mathcal{F}| \sum_{f \in \mathcal{F}} x_{f}^{2}},
$$

where $x_{f}$ denotes the throughput of flow $f \in \mathcal{F}$. Results from Table II show that our proposed FBEB algorithm outperforms the CSP [9] and Idle Sense [10] schemes in both short-term and long-term scenarios.

Furthermore, using the method in [19], we determine the max-min fair data rate allocation for each flow in the 3-hop scenario. We compare our proposed FBEB algorithm with the original IEEE 802.11 MAC, CSP and Idle Sense schemes in terms of how far their utility value differs from the optimal solution. We use the $\alpha$-fair utility function in [20]. Let $g_{\alpha}=$ 

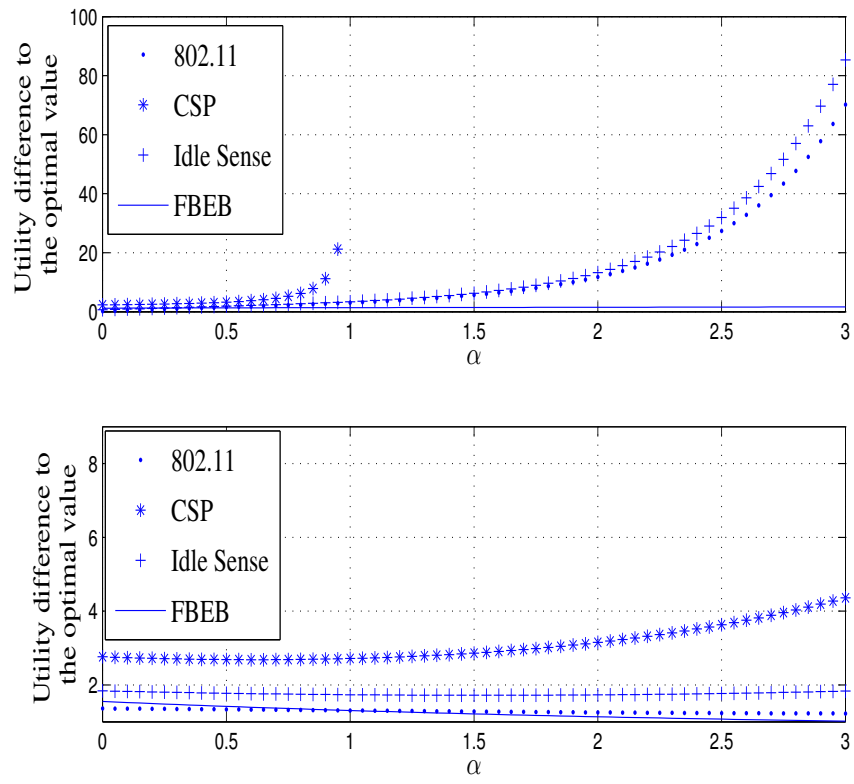

Fig. 5. FBEB algorithm is compared with the original IEEE 802.11 MAC, CSP [9], and Idle Sense [10] in terms of how much their utility value is differed from that of the optimal solution. Our proposed algorithm significantly outperforms the other schemes when $\alpha$ is large. (a): Short-term behavior. (b): Long-term behavior.

$\sum_{f \in \mathcal{F}} f_{\alpha}\left(x_{f}\right)$ where

$$
f_{\alpha}\left(x_{f}\right)= \begin{cases}\log x_{f}, & \alpha=1 \\ (1-\alpha)^{-1} x_{f}^{1-\alpha}, & \alpha \neq 1\end{cases}
$$

Results are shown in Fig. 5. In the y-axis, we have the difference in $\left(g_{\alpha}^{\star}-g_{\alpha}^{\text {method }}\right)$, where $g_{\alpha}^{\star}$ denotes the optimal value and $g_{\alpha}^{\text {method }}$ corresponds to the value obtained from one of the methods (e.g., FBEB, CSP, Idle Sense). Results show that the original MAC performs better when $\alpha$ is small (in which fairness is less important). However, in larger $\alpha$ where the fairness is more important, our proposed algorithm outperforms the other schemes.

\section{CONClusion}

In this paper, we proposed an analytical model to study the flow starvation issue in wireless mesh networks. Since many data transmission sessions in the Internet do not last very long, short-term starvation can dramatically degrade the quality of service provided by the network. We proposed a simple MAC layer technique, called FBEB algorithm, which was shown to alleviate the starvation in short-term sessions. Our proposed FBEB algorithm can increase the channel usage by the nodes farther to the GW about 7 times at the expense of losing $20 \%$ in total throughput. The proposed FBEB algorithm also resulted in a higher fairness index when compared with the CSP and Idle Sense schemes. For future work, we will investigate the performance of our proposed algorithm under different topologies (e.g., grid, random). We will also consider a joint MAC layer and transport layer technique to reduce flow starvation in wireless mesh networks.

\section{ACKNOWLEDGEMENT}

This research is supported by the Natural Sciences and Engineering Research Council (NSERC) of Canada under grant number STPSC 356767-07.

\section{REFERENCES}

[1] M. Garetto, T. Salonidis, and E. W. Knightly, "Modeling per-flow throughput and capturing starvation in CSMA multi-hop wireless networks," in Proc. of IEEE Infocom, Barcelona, Spain, Apr. 2006.

[2] A. Margolis, R. Vijayakumar, and S. Roy, "Modelling throughput and starvation in 802.11 wireless networks with multiple flows," in Proc. of IEEE Globecom, Washington, DC, Nov. 2007.

[3] C. Hua and R. Zheng, "Starvation modeling and identification in dense 802.11 wireless community networks," in Proc. of IEEE Infocom, Phoenix, AZ, Apr. 2008.

[4] G. Anastasi, E. Borgia, M. Conti, and E. Gregori, "IEEE 802.11 ad hoc networks: Performance measurements," in Proc. of Int'l Conf. on Distributed Computing Systems, Providence, RI, May 2003.

[5] M. Garetto, J. Shi, and E. W. Knightly, "Modeling media access in embedded two-flow topologies of multi-hop wireless networks," in Proc. of ACM MobiCom, Cologne, Germany, Aug./Sept. 2005.

[6] H. Y. Hsieh and R. Sivakumar, "IEEE 802.11 over multi-hop wireless networks: Problems and new perspectives," in Proc. of IEEE VTC, Birmingham, Al, May 2002.

[7] K. Xu, M. Gerla, L. Qi, and Y. Shu, "Enhancing TCP fairness in ad hoc wireless networks using neighborhood RED," in Proc. of ACM MobiCom, San Diego, CA, Sept. 2003.

[8] V. Gambiroza, B. Sadeghi, and E. W. Knightly, "End-to-end performance and fairness in multihop wireless backhaul networks," in Proc. of ACM MobiCom, Philadelphia, PA, Sept. 2004.

[9] J. Shi, O. Gurewitz, V. Mancuso, J. Camp, and E. W. Knightly, "Measurement and modeling of the origins of starvation in congestion controlled mesh networks," in Proc. of IEEE Infocom, Phoenix, AZ, Apr. 2008

[10] M. Heusse, F. Rousseau, R. Guillier, and A. Duda, "Idle sense: An optimal access method for high throughput and fairness in rate diverse wireless LANs," in Proc. of ACM SIGCOMM, Philadelphia, PA, Aug. 2005.

[11] A. Raniwala, D. Pradipta, and S. Sharma, "End-to-end flow fairness over IEEE 802.11-based wireless mesh networks," in Proc. of IEEE Infocom, Anchorage, AK, May 2007.

[12] L. Xu, K. Harfoush, and I. Rhee, "Binary increase congestion control (BIC) for fast long-distance networks," in Proc. of IEEE Infocom, Hong Kong, China, Mar. 2004.

[13] J. Shi, T. Salonidis, and E. W. Knightly, "Starvation mitigation through multi-channel coordination in CSMA multi-hop wireless networks," in Proc. of ACM MobiHoc, Florence, Italy, May 2006.

[14] S. Rangwala, A. Jindal, K. Y. Jang, K. Psounis, and R. Govindan, "Understanding congestion control in multi-hop wireless mesh networks," in Proc. of ACM MobiCom, San Francisco, CA, Sept. 2008.

[15] P. Key, L. Massoulie, and D. Towsley, "Path selection and multipath congestion control," in Proc. of IEEE Infocom, Anchorage, AK, May 2007.

[16] A. Khreishah, C. C. Wang, and N. B. Shroff, "Optimization based rate control for communication networks with inter-session network coding," in Proc. of IEEE Infocom, Phoenix, AZ, Apr. 2008.

[17] M. Vutukuru, K. Jamieson, and H. Balakrishnan, "Harnessing exposed terminals in wireless networks," in Proc. of USENIX NSDI, San Francisco, CA, Apr. 2008.

[18] R. Jain, W. Hawe, and D. Chiu, "A quantitative measure of fairness and discrimination for resource allocation in shared computer systems," DEC Research Report DEC-TR-301, Sept. 1984.

[19] Z. Cao and E. W. Zegura, "Utility max-min: An application-oriented bandwidth allocation scheme," in Proc. of IEEE Infocom, New York, NY, Mar. 1999.

[20] J. Mo and J. Walrand, "Fair end-to-end window-based congestion control," IEEE/ACM Trans. on Networking, vol. 8, pp. 556-567, Oct. 2000. 